\title{
Berichtigung zur Arbeit von B. Mueller und W. Y. Ting in Band 11 dieser Zeitschrift: Ist die daktyloskopische Untersuchung als Hilfsmittel zum gerichtlich-medizinischen Aufschluß der Vaterschaft brauchbar?
}

\author{
Von
}

B. Mueller.

Bei dem Referat der Arbeit von Bonnevie: Studies on Papillary Patterns of Human Fingers, ist infolge eines Übersetzungsfehlers leider ein Irrtum unter. laufen. Es heißt aưi Seite 370 in Bd. 11 dieser Zeitschrift: Zur Erforschung des Erbganges benutzt Bonnvie nur Individuen, bei denen sämtliche Finger eine Verschlingung oder wenigstens eine Tendenz zur Verschlingung aufweisen.

Dieser Satz muß einer Berichtigung unterzogen werden. Er ist entstanden infolge fehlerhafter Übersetzung folgender Stelle: Tendency to twisting is notified as a characteristic of the individual when visible in any one of its finger-patterns.

In die deutsche Sprache wörtlich übersetzt: Tendenz zur Doppelschlingenbildung ist als charakteristisches Merkmal für ein Individuum dann anzunehmen. wenn sie (nämlich die Tendenz zur Doppelschlingenbildung) in irgendeinem (nicht jedem) seiner Finger sichtbar ist.

Das Endergebnis unserer Arbeit wird hierdurch nicht beeinträchtigt, da wir schon von uns aus die sich aus der richtigen Utbersetzung ergebende Fragestellung bearbeitet haben.

Frau Professor Bonnevie hat mir num noch persönlich mitgeteilt, daß sie auf Grund neuer embryologischer Studien Doppelschlingen am Daumen für nicht erblich ansieht, daß man also bei Untersuchungen über den Erbgang ein Vorkommen von Verschlingungsmustern am Daumen außer acht lassen muß. Hierdurch werden unsere Ergebnisse vielleicht etwas günstiger gestaltet werden. Nähere Vitteilungen möchte ich mir jedoch bis nach Durchsicht des inzwischen neu gesammelten Materials von weiteren 100 Familien vorbehalten. 